\title{
Localization of Aeroelastic Modes in Mistuned High-Energy Turbines
}

\author{
Christophe Pierre* \\ University of Michigan, Ann Arbor, Michigan 48109 \\ Todd E. Smith $\dagger$ \\ Sverdrup Technology, Inc., Brookpark, Ohio 44135 \\ and \\ Durbha V. Murthy $\ddagger$ \\ University of Toledo, Toledo, Ohio 43606
}

\begin{abstract}
The effects of blade mistuning on the aeroelastic vibration characteristics of high-energy turbines are investigated, using the first stage of the oxidizer turbopump in the Space Shuttle main rocket engine as an example. A modal aeroelastic analysis procedure is used in concert with a linearized unsteady aerodynamic theory that accounts for the effects of blade thickness, camber, and steady loading. High sensitivity of the dynamic characteristics of mistuned rotors is demonstrated. In particular, the aeroelastic free vibration modes become localized to a few blades, possibly leading to rogue blade failure, and the locus of the aeroelastic eigenvalues loses its regular structure when small mistuning (of the order usually present in actual rotors) is introduced. Perturbation analyses that yield physical insights into these phenomena are presented. A powerful but easily calculated stochastic sensitivity measure that allows the global prediction of mistuning effects is developed.
\end{abstract}

\section{Introduction}

$\mathbf{T}$ HE current trend in the design of high-performance propulsion turbomachinery has resulted in systems designed for finite service life. These systems produce high power in compact and light-weight machines, which require stringent safety factors and margins. In this environment, the design engineer is faced with the task of accurately predicting system performance and dynamics. When designing for specific life and reliability goals, the structural dynamic behaviors of the turbomachine are of paramount importance.

The prediction of the dynamics of turbomachine rotors is further complicated by the presence of blade-to-blade differences in structural and material properties. These differences are unavoidable because they arise from manufacturing deviations and in-service degradation. They result in random blade-to-blade variations in natural frequencies, a phenomenon commonly referred to as frequency mistuning. Although most current analyses do not account for mistuning, mistuned rotors may exhibit dynamic characteristics that are vastly different from those of idealized assemblies with identical blades, termed tuned rotors.

In particular, previous studies have demonstrated that mistuning 1) increases the aeroelastic stability of rotors, ${ }^{1.2}$ and 2) results in larger forced response amplitudes. ${ }^{3-5}$ Furthermore, it has recently been shown that mistuning can alter the overall dynamics of rotors with weak interblade coupling in a qualitative fashion. Namely, the equally distributed vibration amplitudes that characterize tuned rotors have been shown

Presented as Paper 91-3379 at the AIAA/SAE/ASME 27th Joint Propulsion Conference, Sacramento, CA, June 24-27, 1991; received Aug. 1, 1991; revision received July 2, 1993; accepted for publication July 27, 1993. This paper is declared a work of the U.S. Government and is not subject to copyright protection in the United States.

*Associate Professor, Department of Mechanical Engineering and Applied Mechanics. Member AIAA.

$\dagger$ NASA Lewis Research Center Group; currently Project Controls Engineer, Construction Operations, Sverdrup Civil Inc., 13723 Riverport Dr., Maryland Heights, MO 63043.

$\ddagger$ Resident Research Associate, Structural Dynamics Branch, NASA Lewis Research Center, Cleveland, OH 44135. Senior Member AIAA. to become localized by mistuning to a few rotor blades, termed rogue blades. ${ }^{6-8}$ This has important implications in that the resulting energy confinement within a few blades indicates a possible cause for rogue blade failure in rotors.

In this article we investigate the effects of blade frequency mistuning on the aeroelastic vibration characteristics of a class of bladed-disk assemblies, namely high-energy turbines with weak aerodynamic coupling between blades. We do not consider structural coupling between blades in this study. This is because previous research ${ }^{6.7}$ has shown that aerodynamic and structural coupling affect the sensitivity to mistuning in the same qualitative fashion. The specific rotor we analyze is the first stage of turbine blades of the high-pressure oxidizer turbopump (HPOTP) in the Space Shuttle main engine (SSME). The SSME rotor was selected because it exhibits many of the characteristics of modern high-performance turbomachinery designs. These include high energy density, low blade aspect ratio, high aerodynamic loading, and advanced superalloy materials. In addition, the SSME turbopump turbines have experienced in-service blade crackings during development and operation. These blades have suffered both low-cycle and high-cycle fatigue, which leads to the presumption that significant dynamic loading may exist. Contrary to previously suggested failure mechanisms (e.g., thermal shock), the present work proposes a theory which is based on the intrinsic dynamic characteristics of mistuned rotors.

This article is organized as follows. Section II presents the structural and aerodynamic models and the formulation of the aeroelastic eigenvalue problem for high-energy turbines. In Sec. III numerical results are presented for the SSME turbine with various levels of mistuning. Efficient perturbation methods that provide insight into mistuning effects are applied in Sec. IV. In Sec. V a stochastic sensitivity measure is developed that allows us to predict the sensitivity of bladed disk assemblies to random mistuning without having to solve mistuned aeroelastic eigenvalue problems. The effectiveness of the sensitivity measure is illustrated by applying it to an advanced unshrouded fan stage. Finally, Sec. VI concludes this article.

There are two main contributions in this work. First, we show the high sensitivity to mistuning and the occurrence of 
localized vibrations for a full model of a real bladed-disk assembly, namely the SSME turbopump turbine. This has important implications for the SSME turbine in particular, and for high-energy turbines with weak interblade coupling in general, because localized vibrations result in higher blade amplitudes and stresses and therefore in shorter fatigue life and possibly rogue blade failure. Second, a fundamental contribution lies in the development of the powerful stochastic sensitivity measure, which allows for the global prediction of mistuning effects based solely upon tuned system information. The sensitivity measure is a generic tool that can be applied to any bladed-disk assembly.

\section{Aeroelastic Formulation}

In this article the bladed disk is modeled as a coupled system of $N$ blades. Each blade's dynamics is described by a single in-vacuum (rotating) natural mode of vibration, say the $n$th natural mode. This simplified representation assumes that the coupling between the natural modes of an individual blade is negligible. Therefore, the rotor equations of motion are a system of $N$ ordinary differential equations, each of which corresponds to an individual blade on the rotor. (Note that we developed a general formulation and a computer program that allow for interactions between various blade modes; however, we found that the blade natural frequencies for the SSME turbopump are so well-separated that intermode coupling is insignificant in the operating range; this justifies the single-mode per blade assumption.)

For simplicity, we assume that the blades are coupled only aerodynamically, and that there is no structural coupling of the blades. Moreover, we are examining the aeroelastic free vibration of the assembly, and include in our model only those aerodynamic forces that are motion-dependent. We also ignore structural damping because this has no qualitative effect on the results. The application of component mode analysis to the $N$-blade assembly yields a set of $N$ homogeneous, linear, ordinary differential equations in the modal amplitudes of the blades. We look for motions such that all the blade coordinates oscillate with the same frequency and/or decay or grow at the same rate. This yields the following aeroelastic eigenvalue problem of order $N$ :

$$
\left[\lambda^{2} M+K-A\left(\omega_{c}\right)\right] \boldsymbol{u}=0
$$

where $\boldsymbol{u}=\left[u_{1}, u_{2}, \ldots, u_{N}\right]^{T}$ is the complex eigenvector of the blade modal amplitudes, where $T$ denotes a transpose; $M$ and $K$ are the real inertia and stiffness matrices, respectively; $A$ is the complex aerodynamic matrix, which depends on the assumed frequency, $\omega_{\omega}$, for the aeroelastic mode of interest ${ }^{10}$; and $\lambda$ is the complex eigenvalue. Since there is no structural coupling, the rotor mass and stiffness matrices are diagonal and of the form

$$
\begin{gathered}
M=I \\
K=\operatorname{diag}\left[\omega_{(n) 1}^{2}, \omega_{(n) 2}^{2}, \ldots, \omega_{(n) N}^{2}\right]
\end{gathered}
$$

where the blade modes have been normalized with a unit modal mass, and $\omega_{(n) j}$ is the $n$th natural frequency of the $j$ th blade (rotating and in a vacuum). For a tuned assembly the diagonal elements of $K$ are identical and equal to $\omega_{(n) 0}^{2}$, the nominal blade frequency squared, i.e., $K=\omega_{(n) 0}^{2} I$. For a mistuned rotor, the individual blade frequencies are generally distinct and the stiffness matrix is diagonal, but not proportional to $I$.

The aerodynamic coupling matrix $A$ is a fully populated complex matrix which is evaluated using the unsteady aerodynamic theory of Verdon and Caspar. ${ }^{11.12}$ Verdon's method is employed to calculate the unsteady forces on the blades due to a particular natural mode of motion for a (tuned) cascade of identical blades. This results in a traveling wave representation of the aerodynamic forces for the tuned cascade. A detailed description of the unsteady force calculation using this theory is given in Refs. 9 and 13 .

The transformation of the aerodynamic influence coefficients between traveling wave coordinates and individual blade, or physical coordinates is defined by

$$
A=E \bar{A} E^{*}
$$

where $\tilde{A}$, the aerodynamic matrix in traveling wave coordinates, is a diagonal matrix of complex elements

$$
\tilde{A}=\operatorname{diag}\left[\tilde{A}_{1}, \tilde{A}_{2}, \ldots, \tilde{A}_{N}\right]
$$

The unitary transformation matrix $E$ is given by

$$
E=\left[e_{1}, \ldots, e_{j}, \ldots, e_{N}\right]
$$

where

$$
\begin{gathered}
\boldsymbol{e}_{j}=\frac{1}{\sqrt{N}}\left[1, e^{i \beta_{j}}, \ldots, e^{i(N-1) \beta_{i}}\right]^{T} \quad \beta_{j}=\frac{2 \pi}{N}(j-1) \\
j=1, \ldots, N
\end{gathered}
$$

where $\beta_{j}$ is the interblade phase angle.

Note that, in general, an iterative procedure is required in order to solve the aeroelastic eigenvalue problem, Eq. (1), because the aerodynamic forces are dependent upon the frequency of vibration. However, for weak aerodynamic coupling, such as exists in high-energy turbines, no iteration is necessary to obtain matched frequency.

The solution of Eq. (1) consists of $N$ pairs of eigenvalues and eigenvectors. For an eigensolution $(\lambda, \boldsymbol{u})$, the blade assembly's motion is given by $\boldsymbol{u} e^{\lambda t}$. The real part of the eigenvalue, $\operatorname{Re}(\lambda)$, determines the aerodynamic damping for the mode, while the imaginary part, $\operatorname{Im}(\lambda)$, is the damped natural frequency of oscillations of the mode. Flutter in a mode occurs when the real part of the eigenvalue is greater than or equal to zero (and if the damped natural frequency of the mode equals the assumed frequency).

For a tuned assembly the matrix $E$ diagonalizes the aeroelastic problem, Eq. (1). This means that the eigenvectors of the system are the columns of $E$, hence, the aeroelastic mode shapes of the tuned assembly are the $e_{j}, j=1, \ldots, N$, given by Eq. (7). For a motion in the $j$ th mode, all blades vibrate with equal amplitudes, but with a constant phase difference $\beta_{j}$ between adjacent blades. We will refer to the modes of the tuned assembly as constant interblade phase angle modes. Physically, these normal mode motions are waves traveling through the assembly with a phase change $\beta_{i}$ at each blade. To each backward traveling wave, $e_{j}$ corresponds a forward traveling wave $\boldsymbol{e}_{N-j+2}$ which has the same number of (traveling) nodal diameters. After diagonalization of Eq. (1) with the similarity transformation defined by $E$, the aeroelastic eigenvalues of the tuned assembly are readily given by

$$
\lambda_{0 j}^{2}=\tilde{A}_{j}-\omega_{0}^{2}
$$

where the subscript $(n)$, representing the $n$th blade mode, is dropped for simplicity.

For a mistuned assembly the constant interblade phase an gle vectors $e_{i}$ do not uncouple the system (1), and thus are not the aeroelastic modes. A numerical or a perturbation solution of the aeroelastic eigenvalue problem is then required. In this work, we assume small random frequency mistuning: the mode shape of each blade is identical, the natural frequency of each blade is a small deviation from the nominal blade frequency, and the frequencies for the individual blades are generated using random numbers from a uniform probability distribution function. The modes of mistuned assemblies are studied in the following sections. 


\section{Mode Localization and Loss of Eigenstructure in the SSME Turbopump Turbine}

The first stage of the SSME turbopump is a rotor consisting of 78 blades equally spaced on the disk. A three-dimensional finite element model is used to calculate the modes of free vibration of individual blades in a vacuum. Figure 1 displays the finite element mesh of one HPOTP turbine blade, along with its first three natural frequencies. In order to account for blade root flexibility, linear springs are included at the surfaces of the firtree lobes. Recall that structural coupling and structural energy dissipation are not included in the model, and that the blades are coupled solely through aerodynamic

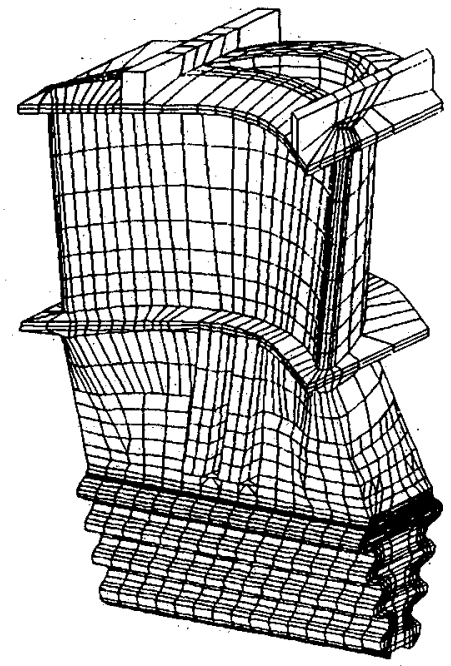

Mode 1 (Bending) $4748 \mathrm{~Hz}$ Mode 2 (Edgewise) $9950 \mathrm{~Hz}$ Mode 3 (Torsion) $16580 \mathrm{~Hz}$

Fig. 1 SSME HPOTP turbine blade finite element model and natural frequencies.

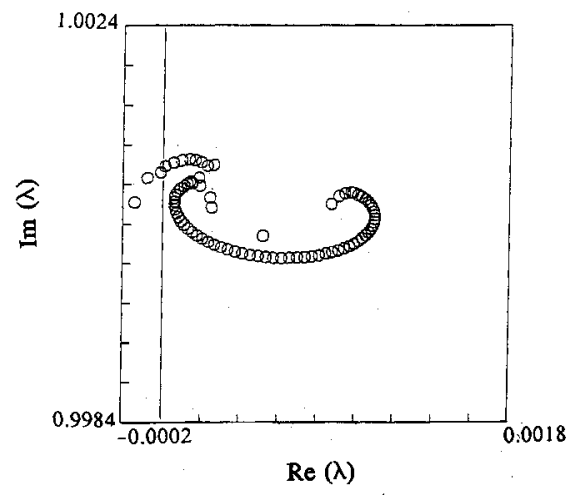

$\varepsilon=0$ (tuned)

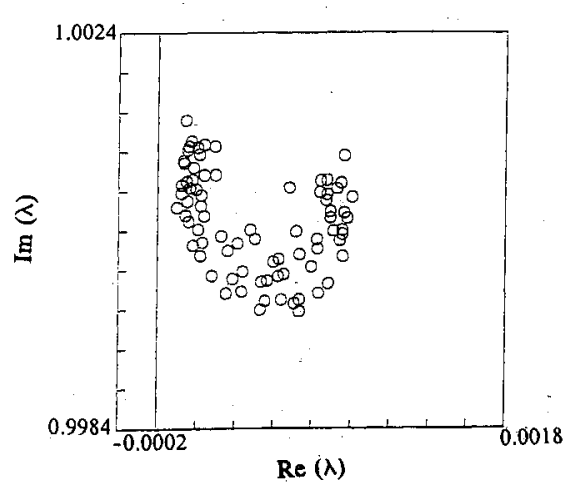

$\varepsilon=0.1 \%$ effects. The unsteady, motion-dependent aerodynamic forces are calculated from a two-dimensional, linearized, unsteady aerodynamic theory applied in axisymmetric strips along the blade span. For details on the structural and aerodynamic models see Refs. 9 and 13 .

We observe in Fig. 1 that the individual blade natural frequencies are well separated. For small damping, the dynamic interactions between the various blade modes due to aerodynamic coupling are negligible. Therefore, it is reasonable to model each blade as a single-degree-of-freedom oscillator for a given natural mode.

We solved the aeroelastic eigenvalue problem for the tuned rotor and for random mistuning of various strengths. Mistuning was measured by its dimensionless standard deviation $\varepsilon$ equal to the standard deviation of the blade natural frequencies divided by the nominal natural frequency. We used a single mistuning pattern for all results. The first three blade modes were considered. For the tuned system, we found that most of the interblade phase angle modes in the second group of modes (corresponding to an edgewise motion of the blades) undergo flutter instability, i.e., have a positive real part of the eigenvalues. This instability can be removed easily by including small structural damping in the model. ${ }^{9}$ No flutter was found in the other groups. Therefore, we focused our investigation of the effects of mistuning on this second group of modes, because it is the least damped. The inlet Mach number for the strips is about 0.27 , and for this second normal mode the reduced frequency (based on chord) is about 2.24 . In all the results presented, the eigenvalues are nondimensionalized with respect to the square of the nominal blade frequency.

\section{A. Scattering of the Root Locus}

The root locus of the 78 aeroelastic eigenvalues in the edgewise mode group is displayed in Fig. 2 for various mistuning
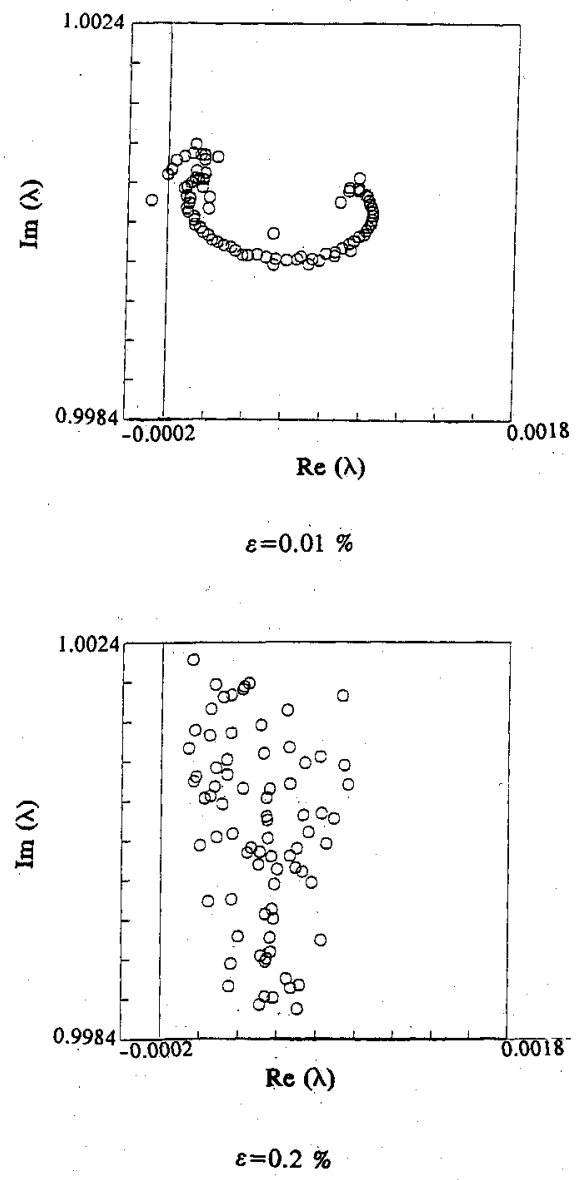

Fig. 2 Root locus, in the complex plane, of the 78 aeroelastic eigenvalues in the edgewise mode group, for various values of blade mistuning. 
values. The imaginary part of the eigenvalues is plotted vs their real part. Note the nearly regular pattern featured by the root locus of the tuned assembly. Also note the narrow range of values spanned by the real parts of the eigenvalues, which indicates weak aerodynamic coupling between blades. As mistuning increases, the regularity of the root locus is gradually lost, and for very small mistuning of standard deviation $0.2 \%$ the locus consists of a constellation of eigenvalues with little discernible pattern. This qualitative change for very small mistuning indicates the high sensitivity of the aeroelastic dynamics of the HPOTP turbine to mistuning. Besides the loss of structure of the root locus, we note that mistuning causes a narrowing of the range of the real parts of the eigenvalues (representing damping), but a stretching of the root locus in the imaginary direction (frequency). In particular, we observe that the real part of the most unstable eigenvalue (that with the largest real part) decreases when mistuning is introduced, thereby confirming the well-known stabilizing effect of mistuning. ${ }^{1,6}$

\section{B. Localization of Aeroelastic Mode Shapes}

The high sensitivity to mistuning detected in the root locus plots is perhaps best illustrated by the changes in the corresponding mode shapes. Figure 3 displays the amplitude pattern of the most unstable aeroelastic mode shape for several mistuning values. As expected, the mode shape of the tuned rotor is a constant interblade phase angle mode and therefore features identical amplitudes for all blades. When mistuning increases, the whole assembly ceases to participate in the motion and the vibration becomes confined to a few of the blades, i.e., the phenomenon of mode localization occurs. The sensitivity of the assembly dynamics to mistuning is extreme: even for a very small mistuning of $0.05 \%$ the blade amplitudes vary widely throughout the rotor, and for $1 \%$ mistuning only four blades participate in the motion to any significant degree! This indicates that the transition from constant interblade phase angle modes to localized modes is very rapid.

In order to illustrate further this dependence of localization on the mode number, Fig. 4 shows the amplitude patterns of 4 of the 78 aeroelastic mode shapes for a given mistuning strength: 2 modes at the extremes of the frequency cluster; namely the lowest and highest frequency modes, and 2 modes

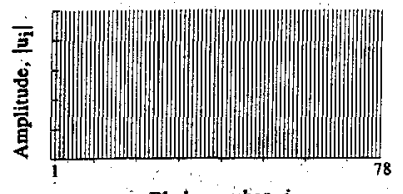

Blade number,

$\varepsilon=0$ (tuned)

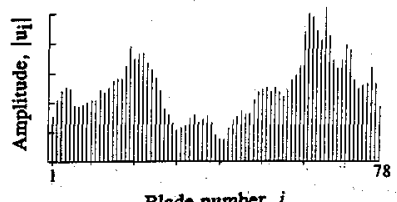

Blade number, $i$

$\varepsilon=0.05 \%$

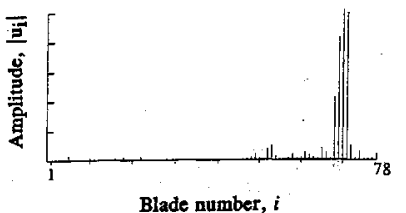

$\varepsilon=0.5 \%$

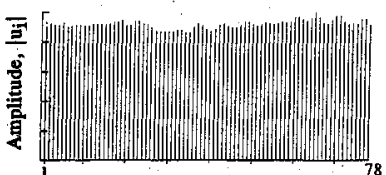

Blade number,

$\varepsilon=0.01 \%$

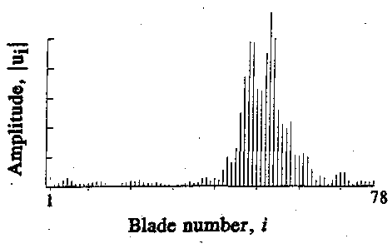

$\varepsilon=0.1 \%$

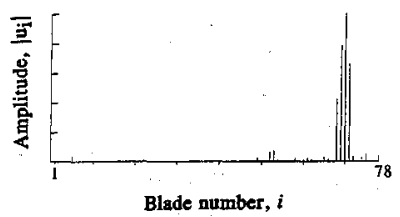

$\varepsilon=1 \%$
Fig. 3 Amplitude pattern of the aeroelastic mode shape associated with the most unstable eigenvalue in the edgewise mode group, for various mistuning strengths. near the middle of the frequency cluster, the least and most stable modes. Observe that the modes with the extreme frequencies are substantially more localized than those near the middle of the frequency band, and hence they are more sensitive to mistuning.

The localization of aeroelastic mode shapes by mistuning has important practical consequences for the HPOTP turbine and possibly other high-energy turbines. First, it means that for such systems, most tuned aeroelastic calculations are probably invalid not only in a quantitative but in a qualitative sense as well. Second, strong mode localization appears to be unavoidable because its onset occurs for mistuning levels that are well below those resulting from typical manufacturing tolerances. For example, for the SSME turbine, testing of several hundred blades led to mistuning standard deviations ranging from 0.5 to $5 \%$ in the various mode groups, while the onset of localization, in the present analysis, is for $\varepsilon=$ $0.01 \%$. Third, the localization of the aeroelastic modes is a potentially dangerous phenomenon, even though mistuning helps stabilize the rotor. This is because the vibrational energy is concentrated in a few of the blades rather than being distributed equally throughout the rotor. Hence, a few blades of the mistuned rotor vibrate with much larger amplitudes than if the rotor were tuned, which results in larger stresses, shorter fatigue life, and possibly blade failure. Localization provides a plausible explanation for the occurrence of blade cracks and single blade failures (rogue blades). Fourth, although our results are only concerned with the free response of the HPOTP turbine, we expect its forced response to be affected by mistuning in a similar way and forced response localization to occur, because the response can be expressed as a combination of motions in the free aeroelastic modes. Such correspondence between free and forced response is indicated by studies of localization in simple models of structurally coupled mistuned assemblies. ${ }^{7.14}$

Note that the above does not necessarily mean that tuned analyses are useless, as they yield conservative "worst case" results and are therefore valuable at the design stage.

\section{Transition from Extended to Localized Modes}

We have seen in Fig. 3 that the transition from constant interblade phase angle (or extended) modes to localized modes is very rapid, occurring primarily between $0-0.5 \%$ mistuning. We also note in Fig. 3 that the region of localization of a mode (i.e., the location of the blades with the largest amplitudes) changes for every mistuning level, until severe localization is reached for $\varepsilon=0.5 \%$. This suggests that the transition from extended to localized modes is complex. In this section we examine this transition region.

Figure 5 displays the loci of the real parts of the $10 \mathrm{most}$ unstable eigenvalues vs mistuning strength. In the region from $0 \%$ to approximately $0.05 \%$ mistuning, the variation of the eigenvalue real parts appears to be nearly parabolic, with all eigenvalues being affected by mistuning in a similar way. This parabolic variation, as opposed to a linear one, indicates the high sensitivity to mistuning. It suggests the use of secondorder perturbation methods to capture this high sensitivity (see Sec. IV). In this parabolic region the mistuned system behaves as a perturbation of a tuned assembly, such that the structure of the root locus is nearly regular and the mode shapes feature similar amplitudes for all blades. The loci of the imaginary parts of the eigenvalues (not shown) displays the same features as in Fig. 5 .

Figures 2 and 3 show that the tuned system characteristics begin to break down for $\varepsilon=0.05 \%$. From $\varepsilon=0.05$ to $0.5 \%$ we observe in Fig. 5 a region where the behavior of the eigenvalues exhibits great complexity. We label this zone the transition region. It is characterized by what appear to be numerous crossings of the loci of the eigenvalue real parts. The irregular shape of the lower envelope of these eigenvalue loci is due to the existence of crossings with the other eigenvalues of the system, which are not shown in Fig. 5. 

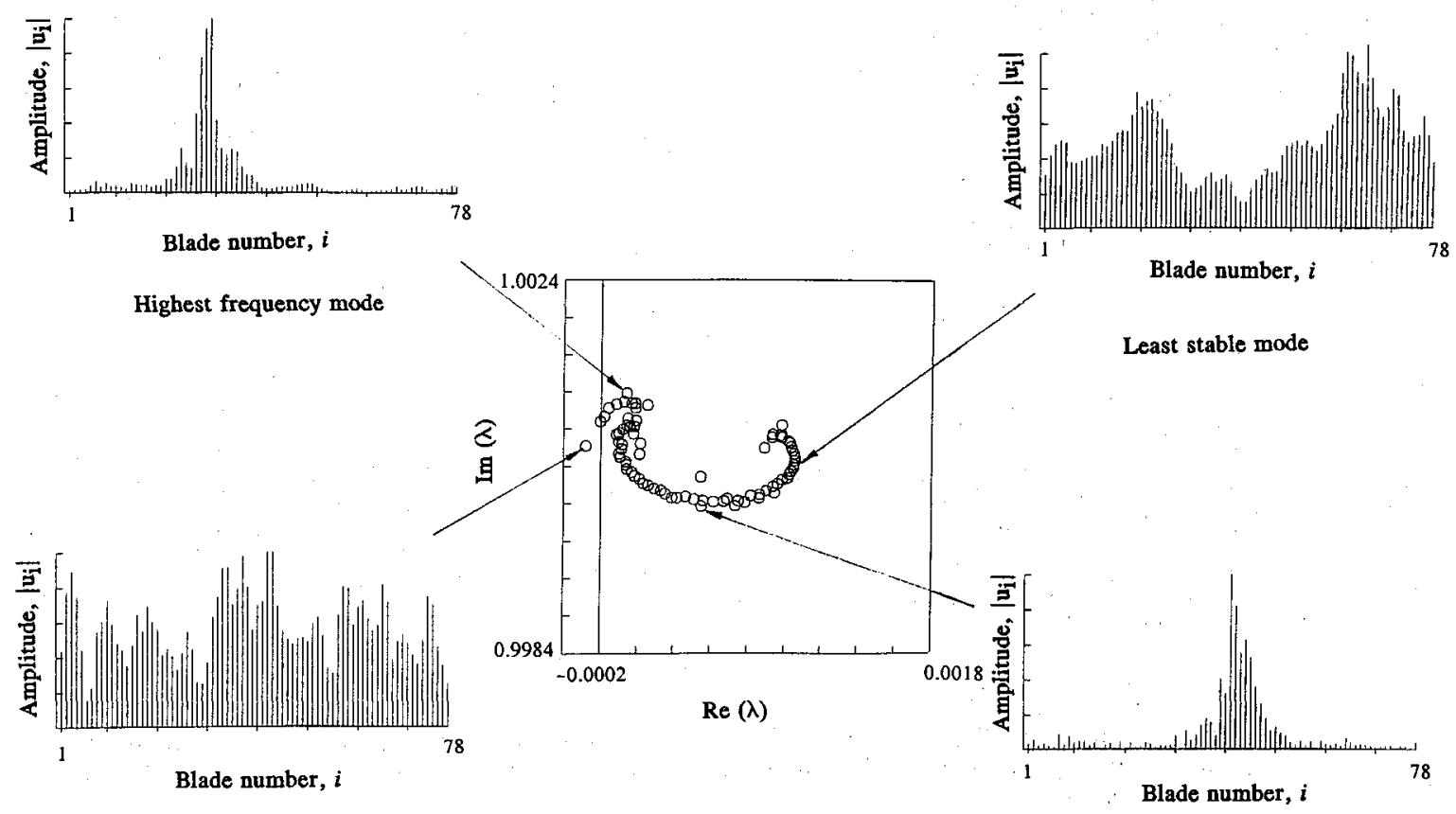

Most stable mode

Lowest frequency mode

Fig. 4 Amplitude patterns of selected aeroelastic mode shapes of an assembly with $\varepsilon=0.05 \%$ mistuning are shown on the associated root locus for the edgewise mode group. The lowest and highest frequency modes and the least and most stable modes are displayed.

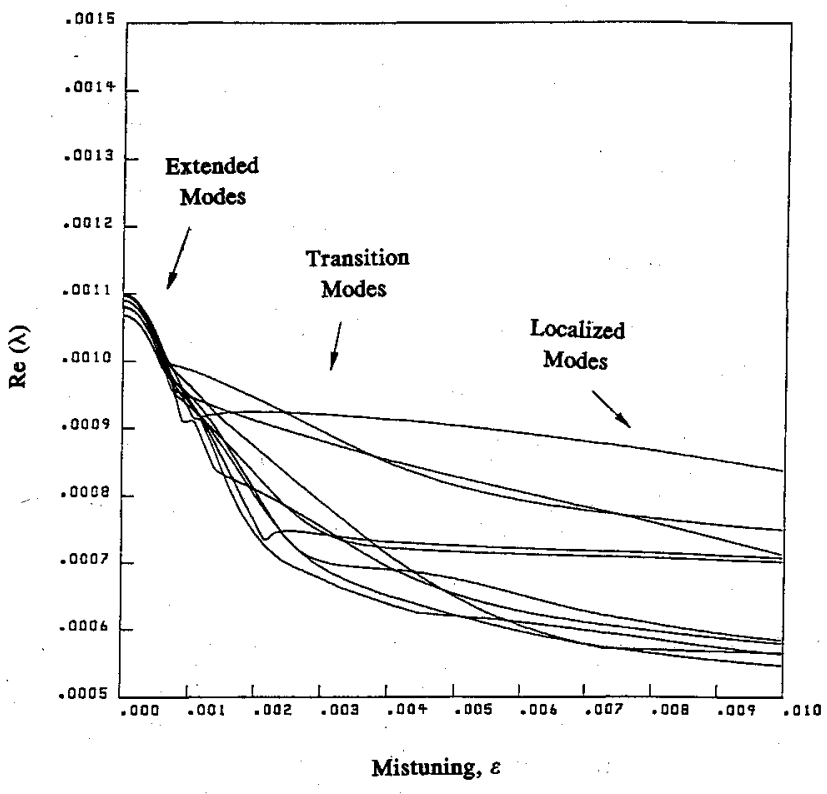

Fig. 5 Loci of the real parts of the 10 least stable eigenvalues in the edgewise mode group, vs mistuning standard deviation.

Although Fig. 5 seems to display eigenvalue crossings, several studies of mode localization in other mistuned periodic systems (e.g., multispan beams and chains of oscillators ${ }^{15}$ ) have shown that eigenvalue loci typically do not cross, but veer away from each other abruptly. Distinguishing between veerings and crossings is difficult unless analytical solutions are available. In the present problem, although we decreased the mistuning increment close to machine accuracy, we were unable to prove that there is a nonzero minimum distance between two eigenvalue loci at their junction. Therefore, we were unable to determine whether veerings or crossings are displayed in Fig. 5. For definiteness we refer to those as "crossings" in the following.

The behavior of the eigenvectors near the eigenvalue loci crossings is of interest. In order to illustrate the change in the mode shapes through a crossing, we selected a nearly simultaneous crossing of three eigenvalue loci in Fig. 5. This triple crossing is displayed in Fig. 6, along with the corresponding mode shapes before and after the crossing.

First, we note that an enlargement of more than two order of magnitudes in mistuning was necessary in order to capture the crossing. This underlines the complexity of the dynamics in the transition region. Second, we observe from Fig. 6 that the modes simply switch positions before and after the crossing, i.e., each mode shape is clearly associated with an eigenvalue loci (here, nearly a straight line) throughout the crossing region. Thus, if we choose to follow a given eigenvalue locus, the character of the associated mode is preserved through the crossing. However, if we choose to track, e.g., the mode with the largest real part (the most unstable mode), this mode changes character every time it goes through a crossing. Since there are numerous crossings in the transition region, we can expect a mode shape to undergo numerous changes as mistuning increases. This is consistent with the observation made in Fig. 3, which shows that the most unstable mode becomes localized at various locations on the rotor as mistuning increases. These changes in the localization region correspond to the crossings with other eigenvalues.

The most striking feature of the transition region is its complexity (recall that only 10 of the 78 eigenvalues are displayed in Fig. 5). Although the bladed disk considered is a linear system, it is apparent from Figs. 5 and 6 that its dynamics are extremely complicated for this range of mistuning, because the mode shapes and eigenvalues are greatly affected by small changes in the mistuning. For example, numerous mode switchings occur. Thus, in practice, it is difficult to predict accurately the modes of assemblies operating in the transition region.

For mistuning greater than $0.5 \%$, the transition to localized modes is nearly complete (see the mode shapes in Fig. 3). This localization region is characterized by fewer eigenvalue loci crossings than in the transition region, and by nearly linear variations of these loci with mistuning. This linear variation is due to the fact that the localized modes can be approximately associated with single decoupled blades, the frequency of which varies linearly with small mistuning (see the perturbation analysis in Sec. IV). This is in contrast with the region 

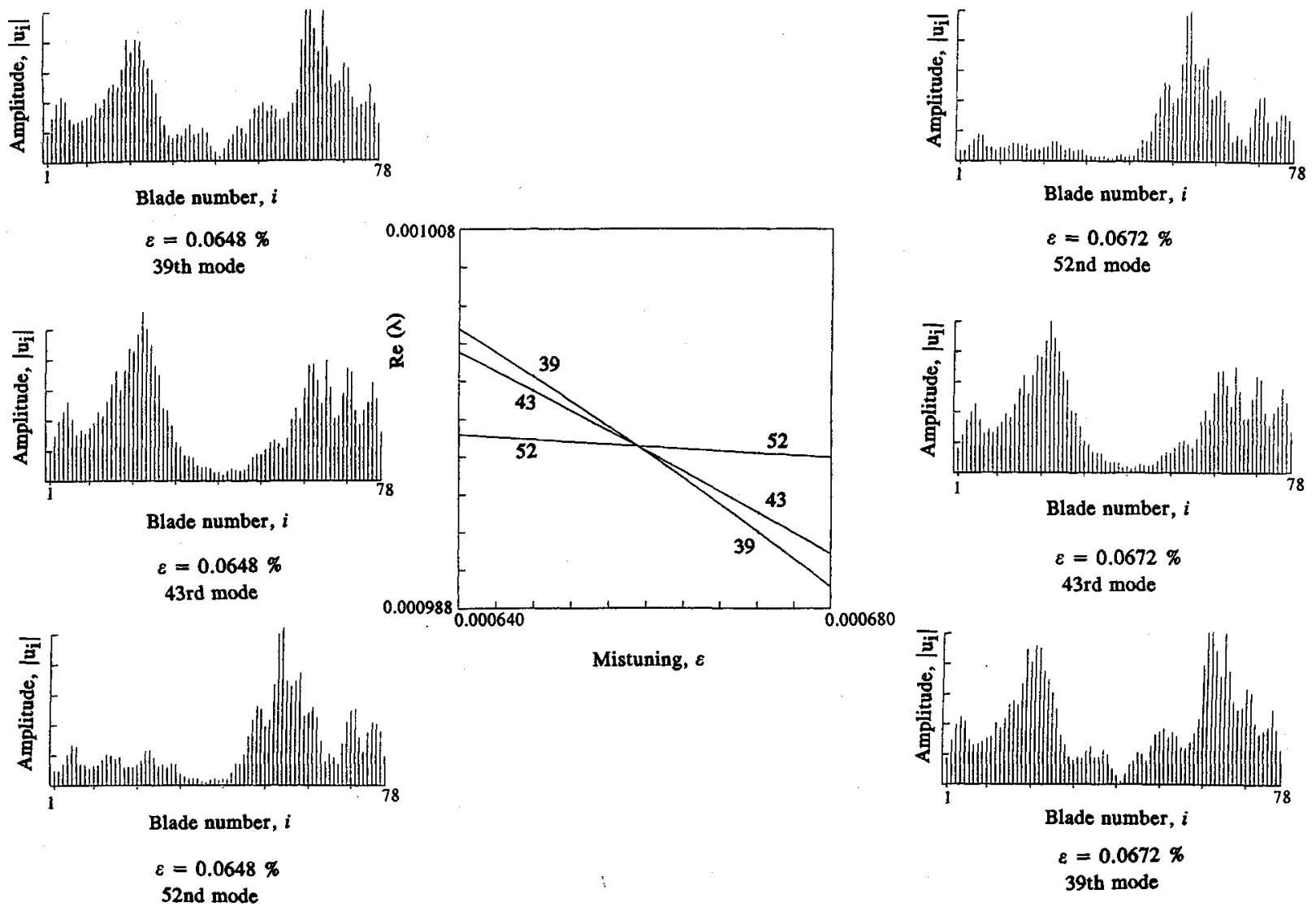

$\varepsilon=0.0672 \%$

43rd mode

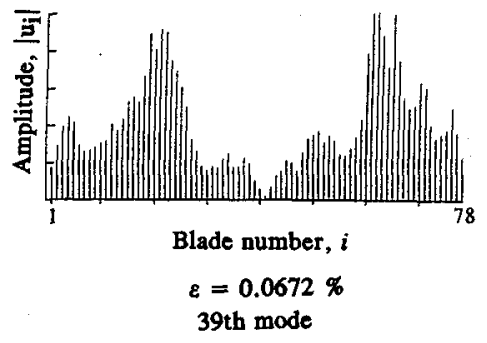

Fig. 6 Crossing of three eigenvalue loci in a small mistuning range (enlargement of Fig. 5). The associated mode shapes before and after the crossing are shown.

of very small mistuning, which features the high sensitivity associated with parabolic variation.

Our results for the HPOTP turbine indicate that severe localization occurs at the mistuning levels measured for the SSME blades (typically between $1-4 \%$ ), and that no modes belong to the transition region. However, structural coupling through the disk or tip shrouds would lower the sensitivity to mistuning and thus extend the transition region. Therefore, it is conceivable that some of the modes of the real HPOTP rotor belong to the transition region and, although very different from extended modes, are not fully localized to a few blades. Moreover, other high-energy turbines may have different parameter values (interblade coupling, number of blades, blade stiffness, etc.), and may feature modes in the transition region. These modes would be difficult to predict accurately, as discussed previously.

\section{Localization of Various Mode Groups}

The aeroelastic modes of the $N$-blade assembly are clustered in groups of $N$ modes, such that each group corresponds to a blade mode. While the results given above focus on the effects of mistuning on the second mode group, it is of interest to compare the sensitivity of the other groups of blade modes and their localization.

The first three normal modes of the HPOTP turbine blade are bending, edgewise, and torsion, respectively. Figure 7 displays the amplitude patterns of the most unstable mode of the assembly in each group. The frequency mistuning pattern, as well as the mistuning strength of $0.5 \%$, are taken to be identical for all three mode groups. We observe in Fig. 7 that although the bending mode is substantially affected by mistuning, it does not become localized. On the other hand, the modes in the edgewise and torsion groups become strongly localized, the latter slightly more than the former. Thus, the bending mode group is much less sensitive to mistuning than the edgewise and torsional mode groups, which feature nearly the same sensitivity. This behavior is explained in the next section.

\section{Perturbation Analyses}

In this section we seek an understanding of the physical mechanisms that govern the dynamics of mistuned assemblies. To that end, we have developed perturbation schemes that predict the high sensitivity to mistuning as well as characterize the degree of localization of the modes. Here we apply this perturbation approach to the HPOTP turbine. For details on the perturbation analyses, see Ref. 6 .

\section{A. Classical Perturbation Approach}

The most natural perturbation procedure to study the dynamics of a mistuned assembly is one that considers the tuned assembly as the unperturbed system, and the blade mistuning as the small perturbation. This approach, which we refer to as the classical perturbation method, yields easily eigenvalue and eigenvector perturbations to any order in the mistuning (first- and second-order results are given below). However, this classical approach is inherently flawed in cases of high sensitivity and localization. This is because choosing the small mistuning as the perturbation parameter requires the eigensolution of the mistuned assembly to be a small perturbation of that of the tuned assembly. Clearly, this is not the case when the phenomena of mode localization and root locus scattering occur, since in this case mistuning causes qualitative (i.e., very large) alterations in the assembly's dynamics. Thus, the classical perturbation analysis cannot capture the drastic changes in the dynamics caused by small mistuning. As we discuss below, however, the mere fact that the technique fails in the presence of localization can be used to predict the high sensitivity to mistuning.

Let us now explore the mechanisms of failure of the classical perturbation method. We denote the unperturbed stiffness matrix of the tuned assembly by $K_{0}$, and the perturbation 

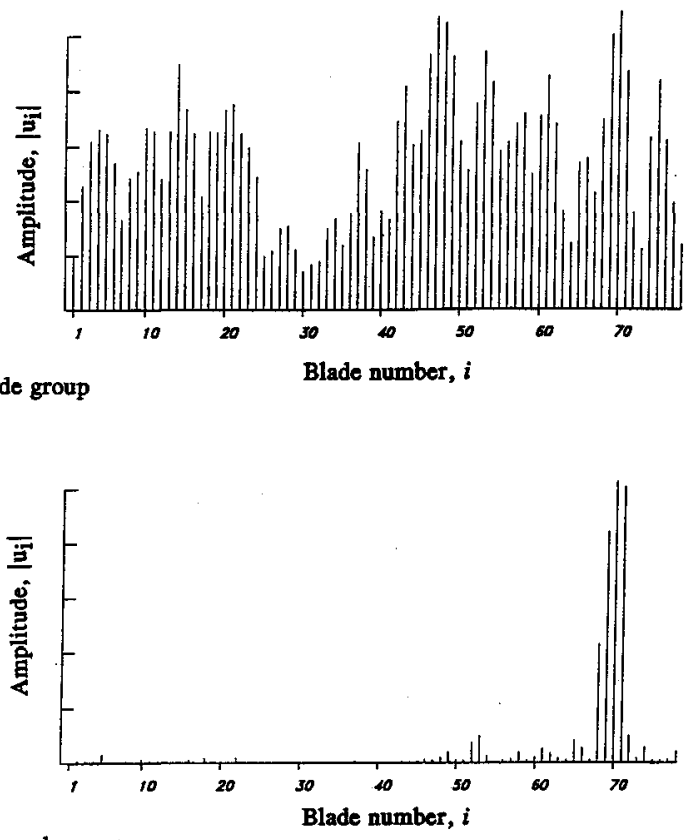

Edgewise mode group

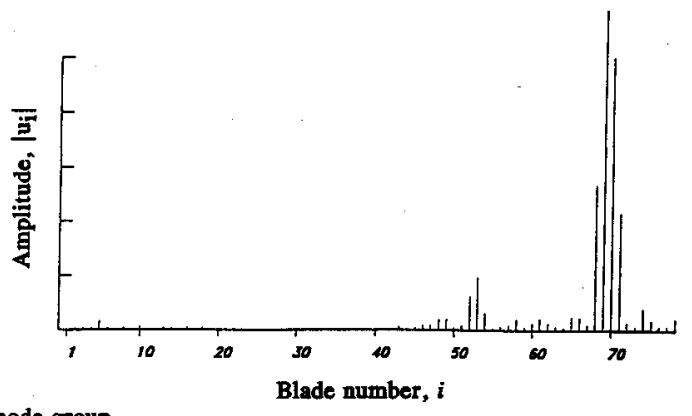

Torsion mode group

Fig. 7 Amplitude pattern of the least stable mode shape in each of the first three mode groups (bending, edgewise, and torsion). In all cases the blade mistuning is $\varepsilon=0.5 \%$.

matrix due to small mistuning by $\delta K$, where the latter is a diagonal matrix of mistunings in the squares of the individual blade frequencies $\delta \omega_{i}^{2}$, such that $\delta \omega_{i} / \omega_{0} \ll 1$ for $i=1, \ldots$, $N$. The stiffness matrix of the perturbed (mistuned) assembly is therefore $K=K_{0}+\delta K$. The modes of the unperturbed system are the interblade phase angle modes given by Eqs. (7) and (8). Those of the perturbed system can be expanded in a perturbation series as

$$
\left.\begin{array}{c}
\lambda_{i}^{2}=\lambda_{0 i}^{2}+\delta \lambda_{i}^{2}+\delta^{2} \lambda_{i}^{2} \\
\boldsymbol{u}_{i}=e_{i}+\delta \boldsymbol{u}_{i}+\delta^{2} \boldsymbol{u}_{i}
\end{array}\right\} \quad i=1, \ldots, N
$$

where $\delta \lambda_{i}^{2}$ and $\delta u_{i}$ (respectively $\delta^{2} \lambda_{i}^{2}$ and $\delta^{2} u_{i}$ ) are first-order (respectively, second-order) terms in the small mistuning.

Applying perturbation theory to the eigenvalue problem, ${ }^{16}$ one can show that the first-order perturbation of the eigenvalues is

$$
\delta \lambda_{i}^{2}=-\frac{1}{N} \sum_{k=1}^{N} \delta \omega_{k}^{2} \quad i=1, \ldots, N
$$

We readily observe that for small mistuning this is always a small term. Hence, the first-order eigenvalue perturbation cannot reveal high sensitivity to mistuning. Moreover, all eigenvalues are shifted by an identical amount and, for a mistuning pattern that averages to zero, the change in the eigenvalues equals zero. Another interesting remark is that the perturbation of the eigenvalue squared is real, therefore mis- tuning, to the first-order, does not affect the stability of the assembly (or very little). Here we reach the seemingly contradictory conclusion that if the average mistuning throughout the rotor is not zero, i.e., if we stiffen or soften all the blades on the average, the flutter boundary remains unchanged! The explanation of this paradox lies in the fact that the assumed frequency that was used for the aerodynamic computations is then no longer valid. If the effect of mistuning with nonzero mean on flutter is sought, the full mistuned eigenvalue problem must be solved in order to determine the correct assumed frequency. These remarks suggest that, although simple and cost-effective, perturbation methods must be used with care in a design environment.

Returning to our main discussion, we now give the secondorder perturbation of the eigenvalues

$$
\delta^{2} \lambda_{i}^{2}=\sum_{\substack{k=1 \\ k \neq i}}^{N} \frac{\left|e_{k}^{*} \delta K e_{i}\right|^{2}}{\lambda_{\overline{0} i}^{2}-\lambda_{0 k}^{2}}=\sum_{\substack{k=1 \\ k \neq i}}^{N} \frac{\left|e_{k}^{*} \delta K e_{i}\right|^{2}}{\tilde{A}_{i}-\tilde{A}_{k}} \quad i=1, \ldots, N
$$

where ${ }^{*}$ denotes complex conjugate and $\mid$ the modulus of a complex quantity. In Eq. (11), the numerator in the summation is a measure of the square of the mistuning (in the frequency squares), and the denominator is the spread among the interblade phase angle aerodynamic coefficients. For the HPOTP turbopump, these aerodynamic coefficients are small because the unsteady aerodynamic forces are small compared to elastic and inertia forces and provide very weak coupling between the blades. Equation (8) shows that the spread in the interblade phase angle aerodynamic coefficients is simply that in the tuned aeroelastic eigenvalues. From Fig. 2, this spread is seen to be about four orders of magnitude smaller than the square of the nominal blade frequency. Thus, if mistuning is of the order of, e.g., $1 \%$, the numerators and denominators in Eq. (11) are of comparable magnitudes and the second-order eigenvalue perturbation is of the order of one, not second-order. The fact that eigenvalue perturbations become large indicates the failure of the perturbation analysis and reveals the high sensitivity of the assembly dynamics to mistuning.

Several remarks are in order. First, the above discussion examines a single term in the summation, Eq. (11). Multiple terms in the summation generally increase the magnitude of second-order perturbation. Therefore we expect the sensitivity to mistuning to increase with the number of blades (this is discussed in Sec. V). Second, although not shown here, the aeroelastic eigenvector perturbations behave similarly to the second-order eigenvalue perturbations, i.e., the sensitivity of the eigenvectors is inversely proportional to the aerodynamic coefficients. This is consistent with the drastic alterations of the mode shapes observed in Fig. 3. Third, although the failure of the perturbation analysis indicates high sensitivity to mistuning, the perturbation results cannot be used to characterize the behavior of the mistuned assembly, as they are qualitatively in error. Fourth, a classical perturbation analysis does not always fail. For example, if mistuning is extremely weak, e.g., $0.01 \%$ for the SSME turbopump, the ratios in Eq. (11) may be sufficiently small for the perturbation expression to be valid. This is also the case if the interblade coupling is strong, such that the aerodynamic coefficients, and thus the denominators in Eq. (11) are not small. In these cases mistuning has a small effect on the assembly's dynamics.

We now have an understanding of the mechanism for high sensitivity to mistuning. It is the closeness of the aeroelastic eigenvalues of the tuned assembly that determines how sensitive the assembly is. This spread in the eigenvalues is governed by the magnitude of the aerodynamic coefficients, and therefore by the strength of the aerodynamic interblade coupling [see Eqs. (8) and (11)]. This mechanism is the same as that identified for assemblies with structural interblade coupling. ${ }^{7}$ In the case of the HPOTP turbine, the interblade 
coupling is very weak and thus the tuned aeroelastic eigenvalues are very close, which results in an extreme sensitivity to mistuning, as shown by the results in the previous section.

For example, we can use the closeness of the tuned eigenvalues to predict the sensitivity in the various groups of modes. For the first group of modes (bending), we found that the spread in the imaginary parts (the frequencies) of the tuned eigenvalues is approximately $0.004 \%$, while the corresponding spread for the second group of modes (edgewise) is only $0.001 \%$. This means, since the distance between eigenvalues is governed by the strength of aerodynamic effects, that the interblade coupling is weaker for the edgewise modes than for the bending modes. Accordingly, the edgewise modes feature a much higher sensitivity to mistuning than the bending modes, as shown by the localization displayed in Fig. 7.

\section{B. Modified Perturbation Method}

While identifying the cause of high sensitivity is useful, it does not provide answers regarding the behavior of the mistuned assembly, viz., the occurrence of mode localization. In order to characterize the dynamics of mistuned assemblies, we have developed a modified perturbation approach described below.

The key idea behind the modified perturbation scheme is to recognize that high sensitivity is caused by small interblade coupling, and therefore the interblade coupling ought to be treated as a perturbation. With this approach, the (modified) unperturbed system is purely structural, consisting of uncoupled mistuned blades in a vacuum. It has distinct natural frequencies (unless two or more blades happen to have the same frequencies, but we do not consider this case here). The (modified) perturbation consists of the small unsteady aerodynamic forces, which provide the interblade coupling. Each normal mode of the unperturbed system features uncoupled oscillations of a single mistuned blade, with all others remaining quiescent. When weak aerodynamic interblade coupling is introduced, the neighboring blades participate in the motion, but do so with small amplitudes because the small coupling is not sufficient to cause a resonance among the slightly different blades. Each mode of the mistuned assembly is a perturbation of the oscillations of a single blade, and thus is localized to that blade or to a small group of blades around it, depending upon the relative magnitudes of the coupling and the mistuning. Examples of localized modes are given in Fig. 3. In summary, the modified perturbation method simply utilizes the fact that, for weak enough interblade coupling or strong enough blade mistuning, the modes of the mistuned assembly are perturbations of the modes of the decoupled mistuned assembly, i.e., of single blade oscillations.

The modified perturbation scheme can be easily implemented by treating the aerodynamic matrix in Eq. (1) as the perturbation; for details see Ref. 6 . The mistuned assembly eigenvalues are given by, to the second-order in the interblade coupling

$$
\lambda_{i}^{2}=-\left(\omega_{i}^{2}+\delta \omega_{i}^{2}\right)+A_{i i}-\sum_{\substack{k=1 \\ k \neq i}}^{N} \frac{A_{k i} A_{i k}}{\delta \omega_{i}^{2}-\delta \omega_{k}^{2}}+\text { H.O.T. }
$$

where the zeroth-, first-, and second-order terms in the interblade coupling are the first three terms on the right side, respectively. Clearly, the effect of the aerodynamic coupling is to modify slightly the assembly eigenvalues from the individual blade frequencies. Also, the second-order term in Eq. (12) indicates that the modified perturbation approach fails when mistuning is too small.

\section{Validity of the Perturbation Schemes}

Figure 8 displays the percentage error between the "exact" numerical eigensolution and the two perturbation results vs the strength of mistuning for the imaginary part of the lowest frequency eigenvalue. We note that for very small mistuning,

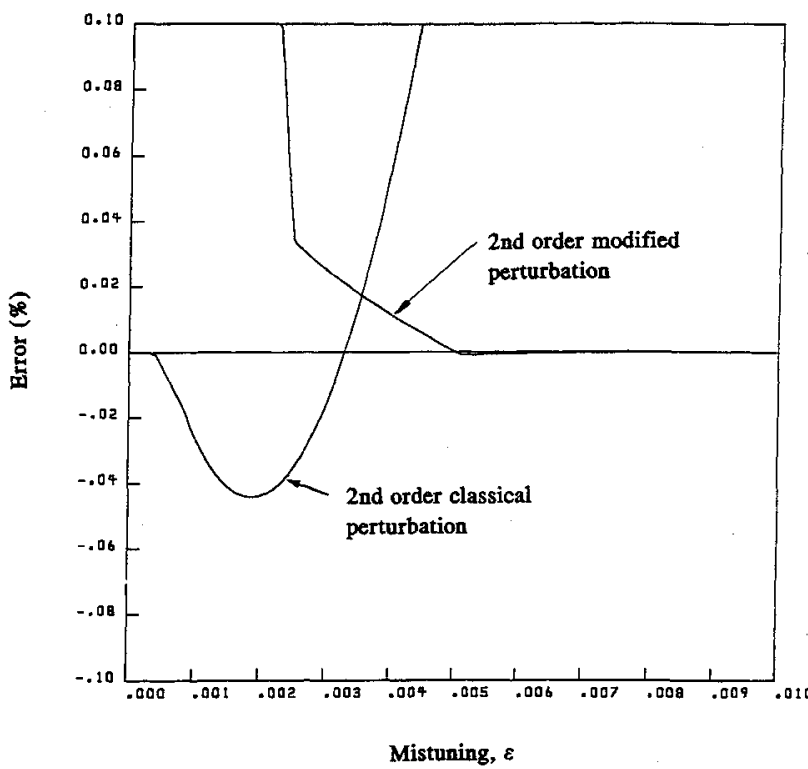

Fig. 8 Comparison of the classical and modified perturbation results with the exact solution. The error from the exact solution is plotted vs the mistuning standard deviation, for the imaginary part of the lowest frequency eigenvalue in the edgewise mode group.

the classical perturbation result agrees well with the exact solution, such that in this region the mistuned assembly behaves as a perturbation of the tuned one. This corresponds to the region of parabolic variation of the eigenvalues in Fig. 5. As mistuning increases, the classical perturbation result diverges rapidly from the exact solution, while the modified perturbation approximation approaches the exact solution. This second zone, where neither perturbation scheme provides a good approximation of the eigensolution, corresponds to the complex transition region. Finally, as mistuning increases to $0.5 \%$, the agreement between the exact and modified perturbation solutions becomes nearly perfect. In this case the mistuned assembly behaves as a perturbation of an assembly of decoupled blades. This corresponds to the localization region characterized by straight eigenvalue loci in Fig. 5. Finally, note that the error of the appropriate perturbation schemes is never greater than $0.05 \%$. This makes the cost-effective perturbation methods accurate analysis and design tools.

As an example of mistuned assembly dynamics prediction, Fig. 9 displays the root locus of the eigenvalues of the HPOTP assembly with $2 \%$ mistuning, by both the modified perturbation scheme and the exact solution procedure. The perturbation method clearly captures the features of the root locus, both qualitatively and quantitatively. For example, the scattering of the locus, as opposed to the regular structure of the tuned locus, is reproduced by the perturbation result. We also note that the exact frequencies (the imaginary parts) are closely matched by the perturbation results. Larger discrepancies occur for the real parts (the damping values), and we believe this is due to the fact that damping is small for all the modes. Finally, we note that one of the eigenvalues (or at least its real part) is not predicted accurately by the perturbation method. We believe this occurs because, in the random mistuning pattern used, two neighboring blades have nearly equal mistuned natural frequencies. In this case the modified perturbation approach fails, see Eq. (12).

\section{Stochastic Measure of Sensitivity}

The perturbation schemes discussed above provide a physical understanding of the sensitivity to mistuning and allow for the analysis of localized modes. In this section we develop a sensitivity measure that will allow the designer to predict, in a very simple way, the effects of mistuning on the various 

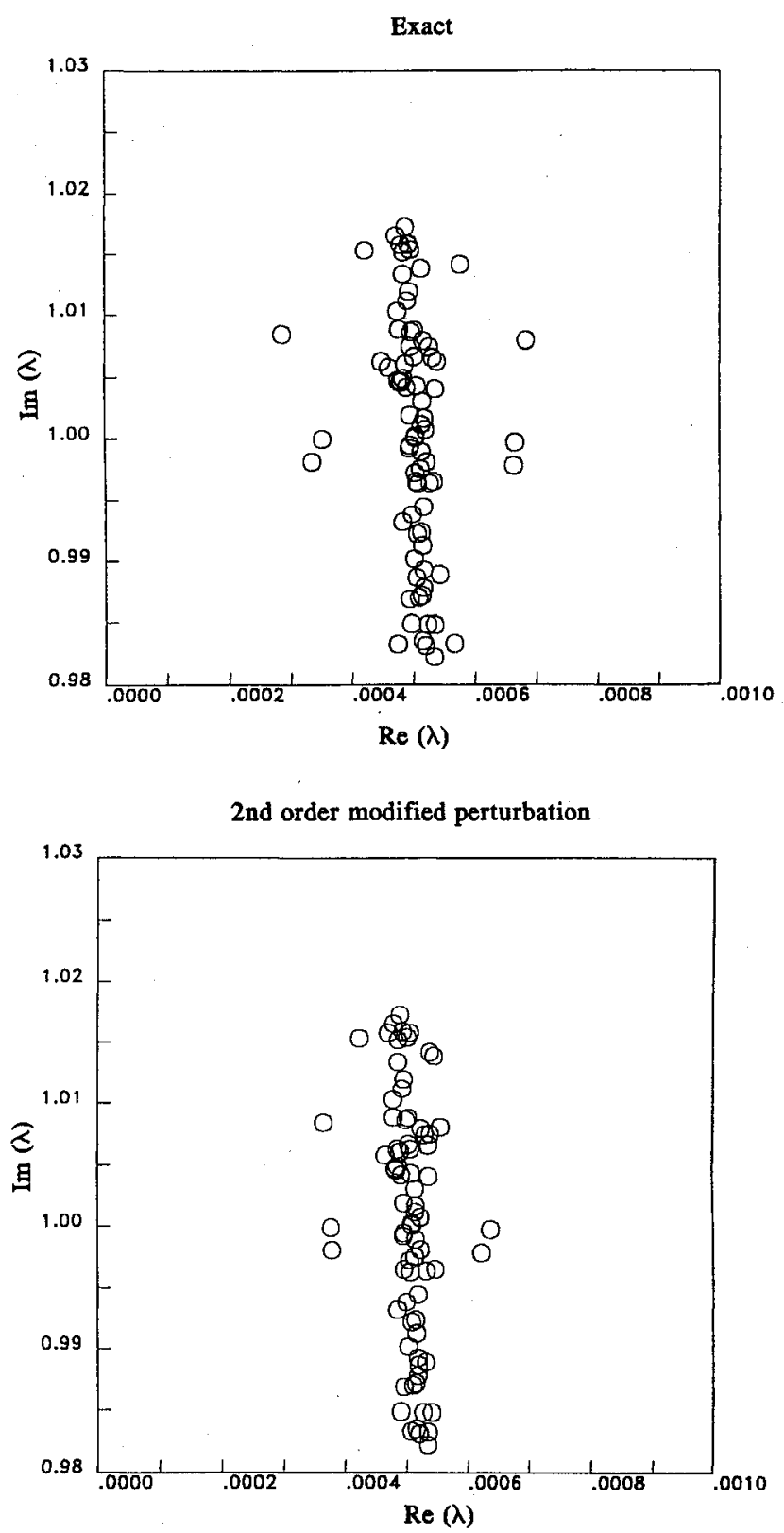

Fig. 9 Root locus of the aeroelastic eigenvalues in the edgewise mode group, by exact solution method and modified perturbation method. The mistuning is $\varepsilon=2 \%$.

aeroelastic modes. Because mistuning is random in nature, a statistical approach is chosen to obtain a compact measure of sensitivity. This measure allows us to predict mistuning effects essentially with a single scalar for each mode. However, a possible drawback of this stochastic measure is that individual realizations of mistuning patterns may result in dynamic behaviors much different from the average behavior predicted by the sensitivity measure (for example, a "sinusoidal" mistuning pattern alters the dynamics much less than a truly random pattern).

The sensitivity measure defined below does not require the mistuned system solution, and therefore, is cost effective. Moreover, since the forced response of mistuned assemblies consists of linear combination of responses in the free modes of vibration, the sensitivity of the aeroelastic modes will provide useful information about that of the forced response. Thus, this measure has the potential to become a valuable design tool.

\section{A. Theoretical Development}

The basic idea is to define the sensitivity of the system by taking the statistical average of the second-order eigenvalue perturbation (here mistuning is the perturbation). This is motivated by the findings in Sec. IV, which showed that the mechanism for high sensitivity, i.e., the closeness of the tuned eigenvalues, is embedded in the second-order perturbation, while the first-order perturbation always remains small. We rewrite Eq. (11) using the expression for the interblade phase angle mode shapes $e_{j}$ given in Eq. (7). After some algebra. we obtain

$$
\begin{aligned}
& \delta^{2}\left(\lambda_{i}^{2}\right)=-\frac{1}{N^{2}} \sum_{\substack{k=1 \\
k \neq i}}^{N} \frac{1}{\tilde{A}_{k}-\tilde{A}_{i}}\left\{\sum_{p} \delta \omega_{p}^{2}\right. \\
& \left.+\sum_{\substack{p, q \\
p \neq q}} \cos \left[\frac{2 \pi}{N}(i-k)(p-q)\right] \delta \omega_{p}^{2} \delta \omega_{q}^{2}\right\}
\end{aligned}
$$

At this point we need to define the mistunings in the frequency squared, $\delta \omega_{p}^{2}, p=1, \ldots, N$, as independent and identical random variables of mean zero and standard deviation $\sigma$. This simply means that the blades are chosen randomly from an (ideally) infinite population. Next, we take the statistical average, over the random mistunings, of the second-order eigenvalue perturbation. This simply yields

$$
\left\langle\delta^{2}\left(\lambda_{i}^{2}\right)\right\rangle=-\frac{\sigma^{2}}{N} \sum_{\substack{k=1 \\ k \neq i}}^{N} \frac{1}{\tilde{A}_{k}-\tilde{A}_{i}} \quad i=1, \ldots, N
$$

because $\left\langle\left(\delta \omega_{p}^{2}\right)^{2}\right\rangle=\sigma^{2}$, and for $p \neq q,\left\langle\delta \omega_{p} \delta \omega_{q}\right\rangle=0$, where \langle\rangle denotes an average.

Now consider the Taylor expansion of the $i$ th eigenvalue in terms of mistuning, Eq. (9), and take its statistical average. Since the first-order perturbation is proportional to mistuning, it averages to zero and we obtain

$$
\left\langle\lambda_{i}^{2}\right\rangle=\lambda_{0 i}^{2}-\frac{\sigma^{2}}{N} \sum_{\substack{k=1 \\ k \neq i}}^{N} \frac{1}{\tilde{A}_{k}-\tilde{A}_{i}} \quad i=1, \ldots, N
$$

This shows that to the second-order, the locus of the average of an eigenvalue vs the mistuning standard deviation is a parabola. The curvature of this parabola determines the sensitivity of the associated aeroelastic mode to mistuning. Hence, we rewrite

$$
\left\langle\lambda_{i}^{2}\right\rangle=\lambda_{0 i}^{2}+S_{i} \sigma^{2} \quad i=1, \ldots, N
$$

where we have defined the stochastic sensitivity measure of the $i$ th eigenmode to mistuning as

$$
S_{i}=-\frac{1}{N} \sum_{\substack{k=1 \\ k \neq i}}^{N} \frac{1}{\tilde{A}_{k}-\tilde{A}_{i}} \quad i=1, \ldots, N
$$

We say a mode features a low or normal sensitivity when the expansion (16) is valid. This occurs when the term $S_{i} \sigma^{2}$ is second-order, and therefore when the sensitivity measure $S_{i}$ is of the order of one. High sensitivity in a mode occurs when $S_{i} \sigma^{2}$ is first-order or larger, implying the failure of the perturbation analysis. This happens when $S_{i}$ is large.

In order to interpret results it is useful to make our sensitivity measure dimensionless. This is achieved first by expressing $S_{i}$ in terms of the dimensionless standard deviation $\bar{\sigma}$, where $\bar{\sigma}=\sigma / \omega_{i}^{2}$. The second step is to divide $S_{i}$ by a representative eigenvalue of the system, such that all sensitivities are referred to 1 rather than to the various $\lambda_{0 i}^{2}$. Here we choose to nondimensionalize $S_{i}$ by the eigenvalue corresponding to the tuned blade frequency squared, $-\omega_{0}^{2}$ (ideally we should divide by $\lambda_{i i}^{2}$ given in Eq. (8), but this would result in a complicated expression for $S_{i}$; moreover, for small aerodynamic coefficients, these two normalizations are nearly 


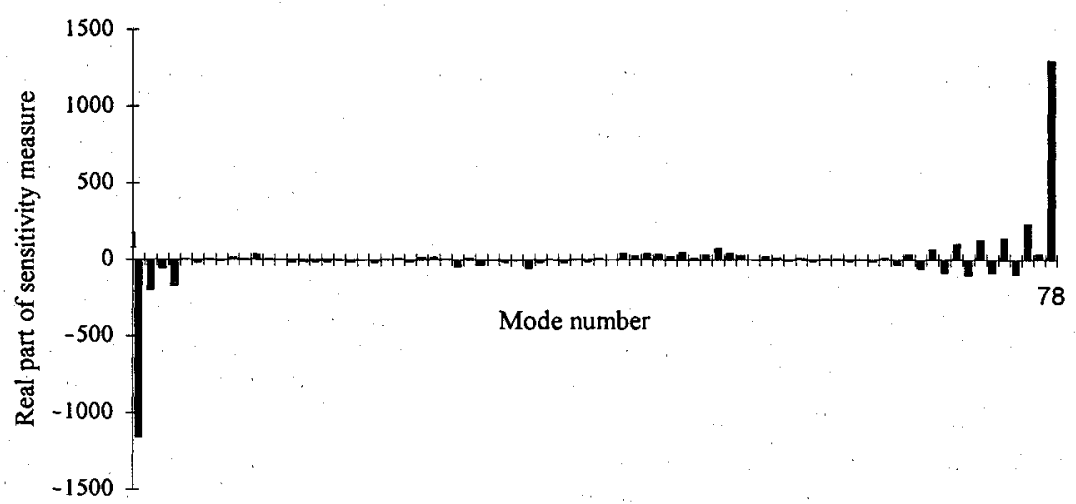

Fig. 10 Real part of the dimensionless sensitivity measure $\tilde{S}_{i}$ (representing the sensitivity of the frequencies) vs mode number. The modes are sorted from the lowest to the highest tuned frequency.
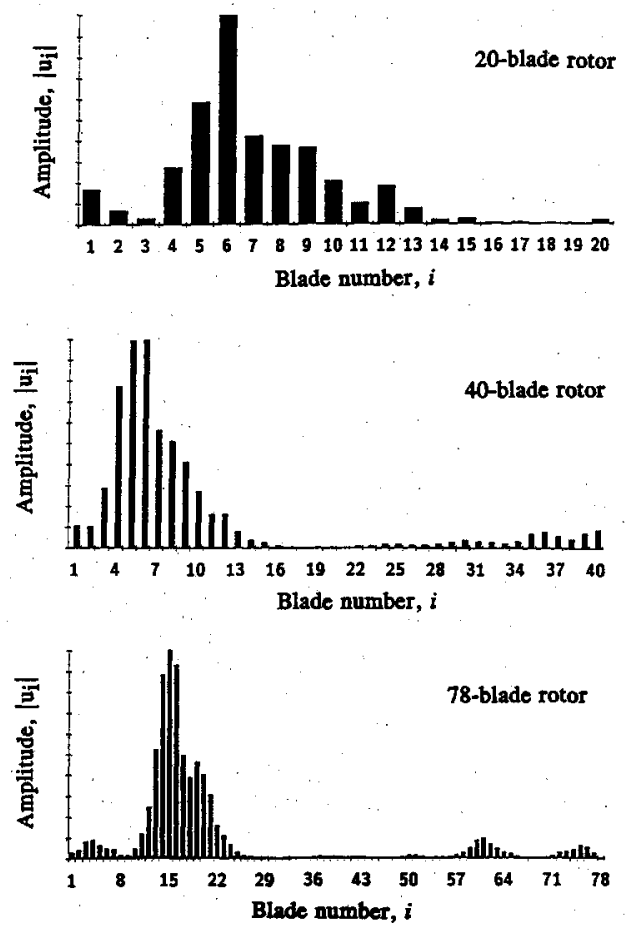

Fig. 11 Amplitude pattern of the lowest frequency mode for various numbers of blades. In all cases the standard deviation of blade mistuning is $\varepsilon=0.63 \%$.

equivalent). The selected normalization yields the dimensionless sensitivity measure:

$$
\bar{S}_{i}=\frac{1}{N} \sum_{\substack{k=1 \\ k \neq i}}^{N} \frac{\omega_{0}^{2}}{\bar{A}_{k}-\tilde{A}_{i}} \quad i=1, \ldots, N
$$

From Eq. (18), we observe readily that the sensitivity measure increases as the aerodynamic interblade coupling decreases and as the number of blades increases. Detailed results are given below.

\section{B. Sensitivity Results}

Here we use our stochastic sensitivity measure in order to reach general results regarding mistuning effects. Note that $\bar{S}_{i}$ is a complex number that characterizes the sensitivity of both the frequency and damping of a tuned mode to mistuning. The damping in a mode, however, is always small (this does not mean it is unimportant), and we have consistently observed that the sensitivity of the frequency is the one that governs mistuning effects. Therefore we focus on the real part of our sensitivity measure.
Since the computation of aerodynamic forces for the SSME turbine is expensive, we used another blade assembly to investigate the effectiveness of $\bar{S}_{i}$ in predicting high sensitivity and localization. This rotor is an advanced unshrouded fan stage, studied earlier. ${ }^{6.17}$ Complete details about the models and the rotor properties can be obtained from these references.

Figure 10 displays the real part (representing frequency) of the sensitivity of the modes of a 78-bladed rotor. The eigenvalues are sorted from the lowest frequency tuned mode to the highest frequency one. The sensitivities of all the modes are very large (much larger than 1 ; note the scale). This predicted high sensitivity is confirmed by the lowest frequency mode of the 78-blade rotor, displayed in Fig. 11 for $\varepsilon=$ $0.63 \%$. This mode exhibits strong localization features. Moreover, Fig. 10 shows that the modes with the lowest and the highest frequencies are much more sensitive to mistuning than those near the middle of the frequency cluster. This is fully consistent with the results presented in Fig. 4 for the SSME turbine, which showed that the least and most stable modes (typically located near the middle of the mode group) are less severely localized than the lowest and highest frequency modes.

Figure 11 displays the lowest frequency mode of this rotor for various numbers of blades, from $N=20$ to $N=78$. We note that while the mode of the 20 -blade assembly is localized to approximately one-half the blades on the rotor, the degree of localization of the same mode increases rapidly with the number of blades. For the 78-blade rotor, only one-sixth of the blades participate significantly in the motion. The corresponding calculated absolute values of the sensitivity measures for real parts of eigenvalues are 249 for $N=20$ and 1167 for $N=78$, and are consistent with the mode shapes in Fig. 11 .

On a final note, we point out that the calculation of the sensitivities for all the modes is a trivial task that requires only the tuned assembly's eigensolution. No solution of the mistuned eigenvalue problem is required. This sensitivity measure could be used effectively as a design tool.

\section{Concluding Remarks}

The main findings of this study are 1) weak aerodynamic coupling causes the aeroelastic characteristics of high-energy turbines to be highly sensitive to small random blade mistuning, and 2) the presence of high sensitivity can be detected by a stochastic sensitivity measure based solely on tuned system information. Besides providing a physical understanding of mistuning effects and being cost-effective, the sensitivity measure developed here has the potential to become a useful tool for the turbine designer.

Upon introduction of small mistuning, the root locus of the aeroelastic eigenvalues loses the regular structure that characterizes the tuned assembly to become randomly scattered, and the constant interblade phase angle mode shapes of the tuned assembly become strongly localized to a few of the 
blades. The occurrence of vibration localization has important consequences for high-energy turbines. Localization results in a confinement of vibrational energy to a few of the blades, and therefore, in a possibly large increase in amplitude for those blades. In turn, stresses are larger and fatigue life is shorter, which can ultimately result in the failure of those few blades.

We did not consider structural coupling between blades in our study. However, previous research ${ }^{6,7}$ has shown that aerodynamic and structural coupling affect the sensitivity to mistuning in the same qualitative fashion. Hence, including structural coupling in our model would increase interblade coupling and lower the sensitivity to mistuning. Let us consider an extreme situation where the structural coupling is 10 times stronger than the aerodynamic one. Since the degree of localization is a function of the ratio of coupling to mistuning, ${ }^{15}$ the assembly with both aerodynamic and structural couplings requires a mistuning 10 times larger than that for the aerodynamic-only system in order to produce the same localization. For the SSME, since $0.1 \%$ mistuning causes strong localization in the aerodynamic-only system, it means that a mistuning of $1 \%$ would be needed. With mistuning estimates typically ranging from 1 to $5 \%$, we conclude that high sensitivity and strong localization would still be observed in this extreme situation. The study of mistuned assemblies with both structural and aerodynamic interblade coupling will be the subject of future research.

\section{Acknowledgments}

This work was supported by the Institute for Computational Mechanics in Propulsion and by NASA Lewis Research Center through Grants NAG3-1163 and NAG3-742 and Contract NAS3-25266. George Stefko was the technical monitor.

\section{References}

'Bendiksen, O. O., "Flutter of Mistuned Turbomachinery Rotors," Journal of Engineering for Gas Turbine and Power, Vol. 106, 1984, pp. 25-33.

'Crawley, E. F., and Hall, K. C., "Optimization and Mechanisms of Mistuning in Cascades," Journal of Engineering for Gas Turbine and Power, Vol. 107, 1985, pp. 418-426.

${ }^{3}$ Ewins, D. J., "Vibration Characteristics of Bladed Disc Assemblies," Journal of Mechanical Engineering Science, Vol. 15, 1973, pp. 165-186.

${ }^{4}$ El-Bayoumy, L. E., and Srinivasan, A. V., "Influence of Mistuning on Rotor-Blade Vibrations," AIAA Journal, Vol. 13, 1975, pp. $460-464$.

${ }^{5}$ Kielb, R. E., and Kaza, K. R. V., "Effects of Structural Coupling on Mistuned Cascade Flutter and Response," Journal of Engineering for Gas Turbine and Power, Vol. 106, 1984, pp. 17-24.

${ }^{6}$ Pierre, C., and Murthy, D. V., "Aeroelastic Modal Characteristics of Mistuned Blade Assemblies: Mode Localization and Loss of Eigenstructure," AIAA Journal, Vol. 30, No. 10, 1992, pp. 24832496.

${ }^{7}$ Wei, S.-T., and Pierre, C., "Localization Phenomena in Mistuned Assemblies with Cyclic Symmetry. Part I: Free Vibrations," Journal of Vibration, Acoustics, Stress, and Reliability in Design, Vol. 110, 1988, pp. 429-438.

${ }^{8}$ Valero, N. A., and Bendiksen, O. O., "Vibration Characteristics of Mistuned Shrouded Blade Assemblies," Journal of Engineering for Gas Turbine and Power, Vol. 108, 1986, pp. 293-299.

'Smith, T. E., "A Modal Aeroelastic Analysis Scheme for Turbomachinery Blading," NASA CR 187089, 1991.

${ }^{10}$ Murthy, D. V., and Kaza, K. R. V., "A Computational Procedure for Automated Flutter Analysis," Communications in Applied Numerical Methods, Vol. 5, 1989, pp. 29-37.

"Verdon, J. M., and Caspar, J. R., "Development of a Linear Unsteady Aerodynamic Analysis for Finite-Deflection Subsonic Cascades," AIAA Journal, Vol. 20, 1982, pp. 1259-1267.

${ }^{12}$ Verdon, J. M., and Caspar, J. R., "A Linearized Unsteady Aerodynamic Analysis for Transonic Cascades," Journal of Fluid Mechanics, Vol. 149, 1984, pp. 403-429.

${ }^{13}$ Smith, T. E., "Aeroelastic Stability Analysis of a High-Energy Turbine Blade," AIAA Paper 90-2351, 1990.

${ }^{14}$ Wei, S.-T., and Pierre, C., "Localization Phenomena in Mistuned Assemblies with Cyclic Symmetry. Part II: Forced Vibrations," Journal of Vibration, Acoustics, Stress, and Reliability in Design, Vol. 110,1988 , pp. $439-449$.

${ }^{15}$ Pierre, C., "Mode Localization and Eigenvalue Loci Veering Phenomena in Disordered Structures,"Journal of Sound and Vibration, Vol. 126, 1988, pp. 485-502.

${ }^{16}$ Courant, R., and Hilbert, D., Methods of Mathematical Physics, Volume 1, Interscience, New York, 1953.

${ }^{17} \mathrm{Kaza}, \mathrm{K} . \mathrm{R}$. V., and Kielb, R. E., "Flutter of Turbofan Rotors with Mistuned Blades," AIAA Journal, Vol. 22, 1984, pp. $1618-$ 1625 . 